attempt to sort out what currently is known and not about hazards of ENMs.

Methods The scientific literature from 2000-2017 was assessed using keywords and environmental scanning techniques to capture information about the health effects of ENMs. The focus will be on high volume ENM.

Result The extent of research health effects that have been identified for various high volume ENMs will be described. While many nanomaterials have been developed there are a limited number that are widely used in commerce. Common determinants of toxicity will be identified.

Discussion Assessing the hazard potential of ENM is a complex task since there are so many combinations of physiochemical parameters that may lead to ENMs having differential toxicity. The implications of this will be discussed as well the research needed to address the hazard potential of engineered of nanomaterials that are or could be in commerce.

\section{AIR PNEUMO: AN ACADEMIA-BASED QUALITY ASSURANCE OF PHYSICIANS' PROFICIENCY IN READING CHEST RADIOGRAPHS OF PNEUMOCONIOSIS}

Narufumi Suganuma. Department of Environmental Medicine, Kochi Medical School, Kochi University, Nankoku, Japan

\subsection{6/oemed-2018-ICOHabstracts.34}

Introduction Early detection of the disease, by routine standard chest X-ray, is one of the essential measures for secondary prevention. ILO has provided the guideline for radiographs reading for pneumoconioses according to the ILO classification to support the medical screening test and clinical purposes. The aim of this article is to describe the history and concept of the AIR Pneumo.

Methods Asian Intensive Reader of Pneumoconioses (AIR Pneumo) is a quality assurance program to train and certify physicians who works for prevention of pneumoconises, which is one of the major occupational health problems worldwide. The system is useful for epidemiological research, screening and surveillance of high-risk workers, diagnosis and compensation. Because of variability of reading by physicians, some organisation, e.g., US NIOSH, has developed the certification testing, called B -reader.

Result Since its first Bangkok workshop in 2006, AIR Pneumo has conducted 17 workshops 6 times in Thailand, 4 times in Brazil, twice in Japan, and one in each in Philippines and India. As Bangkok workshops invited international participants, most of physicians from ASEAN countries and D.R. Congo has attended the workshops.

Discussion The classification system holds its uniqueness in possessing standard radiographs that show profusion 0 to 3 for each types of small opacities, large opacities and pleural abnormalities. By using standard radiograph side-by-side to the subject radiographs that physicians are reading and classifying, higher inter reader agreement can be achieved. Understanding and using the classification properly demands certain amount of training. In accordance with WHO/ILO Global Programme for Elimination of Silicosis, ILO has been encouraging GPES participating nations to educate physicians to increase proficiency of reading radiographs of pneumoconioses. The AIR Pneumo, an academia based quality assurance of physicians proficiency in reading pneumoconiosis radiographs is sustainable approach with active involvement of local experts in GPES participant nations.
39 APPLYING HUMAN FACTORS TO PROMOTE A POSITIVE SAFETY CULTURE

Anna-Maria Teperi. Finnish Institute of Occupational Health, Helsinki, Finland

\subsection{6/oemed-2018-ICOHabstracts.35}

Introduction Safety management has traditionally been based on technical solutions and regulations. Although these traditional safety methods are still needed, they do not necessarily raise real safety levels. A prerequisite for improving safety is proper safety management, implemented by people who create and maintain safety through vigilance, competence, communication and group work, if the organisational structures around them support this human success. In this review, I summarise the research and development processes implemented in 2000-2017 to improve the safety, efficiency and well-being of organisations, from the aspect of human factors (HF) with safety critical domains.

Methods In 2000-2017, we conducted interviews, questionnaires and interventions among operative personnel, management and experts in the nuclear industry and the aviation, railway, and maritime sectors. Both business and authorities were represented. The HF tool was designed and modified for each field and the user experiences are presented here. The HF tool consists of a single tool, material for HF training, techniques for analysing cases at work, and proceedings for corrective actions. Its aim is to promote a positive safety culture.

Result The HF interventions helped the organisations handle incidents more transparently, giving way to more open discussions on demanding situations at work. The HF tool became more of a philosophy, with which to highlight and understand human performance at the individual, work, group and organisational level, also taking successes into account. It helped concreticise cognitive, work and organisational psychology as a facilitator of safety.

Discussion The HF tool promoted Safety-II thinking, which has recently been actively discussed as a safety paradigm shift, but has lacked the concrete tools to transform it from scientific debate to practice.

\section{6TH EUROPEAN WORKING CONDITIONS SURVEY: JOB QUALITY IN EUROPE}

Agnès Parent-Thirion, Isabella Biletta, Jorge Cabrita, Oscar Vargas, Greet Vermeylen, Alksandra Wiczynska, Mathijn Wilkens. Eurofound, Dublin, Ireland

\subsection{6/oemed-2018-ICOHabstracts.36}

Introduction The Union and Member states shall have as their objectives improved living and working conditions (article 151 of the TFEU). More and better job is an important policy objective for the European Union.

Methods Statistical analysis of the 6th European Working Conditions Survey. In 2015, the Sixth European Working Conditions Survey interviewed almost 44000 workers in 35 European Countries. Result Seven job quality indices are produced which at the level of the job, gathers these characteristics of work and employment that have been associated in positive or negative with health and wellbeing of workers. The job quality indices are: physical environment, social environment, work intensity, working time quality, skills and discretion, prospects and earnings. They are presented and discussed. Each index is associated with a positive experience of working life in health and well being, work life balance, engagement and motivation, financial security. The job quality indices are pooled together 